\section{Paving possibilities}

New Phytol. http://doi.org/cvt5 (2018).

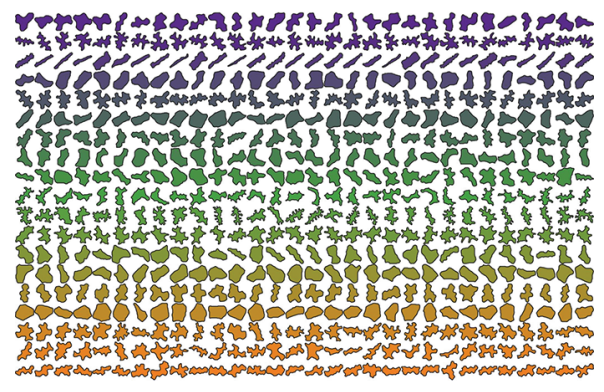

Credit: ROZA V. VOFELEY AND SIOBHAN

BRAYBROOK, UCLA

Plant leaves come in many shapes and sizes, but what of the cells from which they are made? Is the diversity of leaves created by the assembly of essentially similar cells, or are the cells themselves similarly diverse? Siobhan Braybrook of the University of California, Los Angeles; Madelaine Bartlett of the University of Massachusetts, Amherst; and colleagues have addressed this question by analysing the shapes of leaf epidermal pavement cells across a large range of phylogenetic groups.

The researchers collected adult leaves from the Botanic Garden of the University of Cambridge and the University of Massachusetts Amherst Natural History Collection, covering 278 taxa. They took epidermal peels or replicas from the abaxial and adaxial surfaces of these leaves or, failing that, the epidermal cells were released by maceration. The leaf preparations were stained and imaged using light microscopy. The outlines of the cells were traced and their shapes characterised by a combination of measures, such as absolute area, aspect ratio, circularity and solidity. The dataset thus assembled demonstrates the multitude of shapes adopted by plant epidermal cells and will doubtless form the basis for many studies in the future.

The overall picture is of a wider diversity of shapes within groups of plants than between them, but there are also some general trends. For example, gymnosperms and monocots tend to lower aspect ratio longer and thinner - cells than ferns and eudicots. Also, cells on the abaxial surface of a leaf have, in general, more undulating edges than their adaxial counterparts. This property, low solidity, had been considered a distinctive feature of pavement cells, mainly due to studies in Arabidopsis and maize. However, these two model species are outliers in their respective plant groups (eudicots and monocots) having solidity scores closer to the median for ferns than for the generally smoother sided cells of the other plant groups; a reminder of the need to study both model and non-model organisms.

\section{Chris Surridge}

Published online: 2 November 2018 https://doi.org/10.1038/s41477-018-0310-y 